\title{
Die Whirlpool-Dermatitis - eine Sonderform der gramnegativen Follikulitis
}

\section{Whirlpool Dermatitis - A Special Type of Gram-Negative Folliculitis}

Autoren

Institute

\section{P. Nenoff ${ }^{1}$, W. Handrick ${ }^{2}$, J. Herrmann ${ }^{1}$, H.-C. Wenzel ${ }^{3}$}

Laboratorium für medizinische Mikrobiologie, Mölbis

Institut für Medizinische Diagnostik Oderland, Frankfurt/Oder

Hautarztpraxis in Naunhof

\section{Bibliografie}

DOI 10.1055/s-0028-1119608

Online-Publikation: 9.3.2009

Akt Dermatol 2009; 35:

184-185 @ Georg Thieme

Verlag KG Stuttgart · New York ISSN 0340-2541

Korrespondenzadresse

Prof. Dr. W. Handrick

Institut für Medizinische

Diagnostik

Am Kleistpark 1

15230 Frankfurt/Oder

w.handrick@

institut-oderland.de

\section{Zusammenfassung \\ $\nabla$}

Ein 45-jähriger Mann erkrankte an einer Follikulitis durch Pseudomonas aeruginosa. In den folgenden Monaten kam es zu zwei Rezidiven.

\section{Einleitung \\ $\nabla$}

Die Whirlpool-Dermatitis ist ein Krankheitsbild, das zunehmend häufiger beobachtet wird. Dennoch kennen viele Ärzte diese Erkrankung nicht, was zu einer Verzögerung bei der Stellung der Diagnose führen kann. Wir möchten mit folgender Kasuistik nochmals auf diese Problematik hinweisen.

\section{Kasuistik \\ $\nabla$}

Ein 45-jähriger Mann stellte sich wegen entzündlicher Hauteffloreszenzen beim Arzt vor.

Die klinische Untersuchung ergab folgenden Befund: rot-livide, kaum juckende, etwas schmerzende Follikel-assoziierte Papeln und Knoten sowie einzelne Pusteln am Körperstamm. Die Effloreszenzen hatten einen Durchmesser von $0,5-2 \mathrm{~cm}$, waren zentral erhaben und zeigten periphere Infiltrationen ( $\bullet$ Abb. $\mathbf{1}$ a und $\mathbf{b}$ ).

Die kulturelle Untersuchung eines Abstrichs von einer Hauteffloreszenz zeigte massives Wachstum von Pseudomonas aeruginosa. Die histologische Untersuchung eines Biopsats ${ }^{1}$ ergab: flachverruziforme Epidermishyperplasie. Subepithelial Reststrukturen von Haarbestandteilen mit umgebender, teilweise granulozytenreicher Entzündung. Kein Nachweis von Pilzelementen mittels PAS-Färbung. PCR: Pseudomonas aeruginosaDNS nachweisbar.

${ }^{1}$ Wir danken Herrn PD Dr. Th. Mentzel und seinen Mitarbeitern von der Dermatohistologischen Gemeinschaftspraxis Friedrichshafen.
Keimquelle war offensichtlich der Pool im Haus des Patienten. In einer kurzen Literaturübersicht werden einige wichtige Aspekte der WhirlpoolDermatitis durch Pseudomonas aeruginosa dargestellt.
Diagnose: Mit Follikeldestruktion einhergehende, zum Teil granulozytenreiche Follikulitis/Perifollikulitis. In Verbindung mit den klinischen Angaben ist eine Pseudomonas aeruginosa-bedingte Whirlpool-Dermatitis durchaus möglich.

Der Patient wurde zunächst mit FusidinsäureCreme behandelt. Es kam zu keiner Besserung. Nach Vorliegen o.g. Befunde erfolgte eine Therapie mit Ciprofloxacin $(2 \times 250 \mathrm{mg}$ tgl. p.o. über 5 Tage). Die Dermatitis bildete sich komplett zurück. 2 Monate später traten die gleichen Hauteffloreszenzen erneut auf. Auch jetzt erwies sich eine 5-tägige Ciprofloxacin-Therapie als wirksam. 8 Monate später kam es zu einem zweiten Rezidiv. Auch jetzt war die Ciprofloxacin-Gabe erfolgreich.

Als wahrscheinlichste Infektionsquelle konnte letztendlich der im Haus des Patienten befindliche Pool erkannt werden, in dessen Wasser Pseudomonas aeruginosa mit einer Keimzahl von 1 Mio $\mathrm{KbE} / \mathrm{ml}$ nachweisbar war.

\section{Diskussion \\ $\nabla$}

Die Whirlpool-Dermatitis ist eine typischerweise durch Pseudomonas aeruginosa hervorgerufene Follikulitis. Sie betrifft überwiegend gesunde Menschen nach Aufenthalt in öffentlichen Whirlpools, Heißwasser-Becken oder Schwimmbädern.

Oft kommt es dabei zu Fallhäufungen, wodurch die Diagnostik meist beschleunigt wird.

Eine Sonderform der Whirlpool-Dermatitis ist das Hot-Foot-Syndrom, das überwiegend bei jün- 


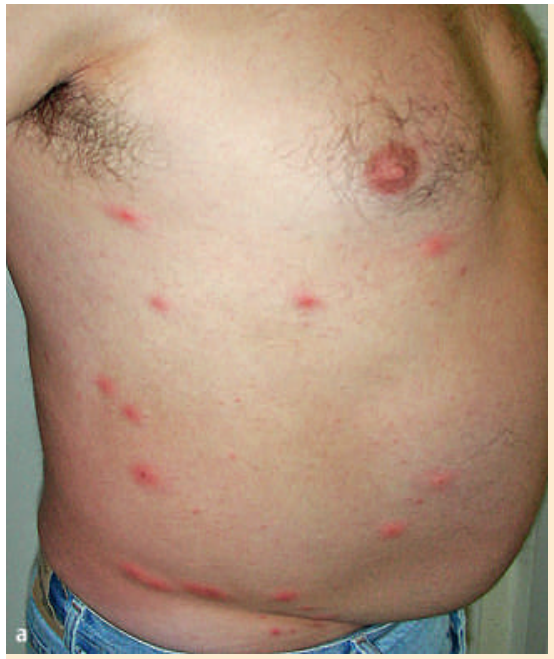

Abb. 1 a, b 45-jähriger Patient: am Körperstamm livid-rote, kaum juckende, etwas schmerzende, follikulär gebundene polymorphe Papeln, Pusteln und Knoten, 0,5 bis $2 \mathrm{~cm}$ Durchmesser, zentral erhaben, peripher infiltriert.

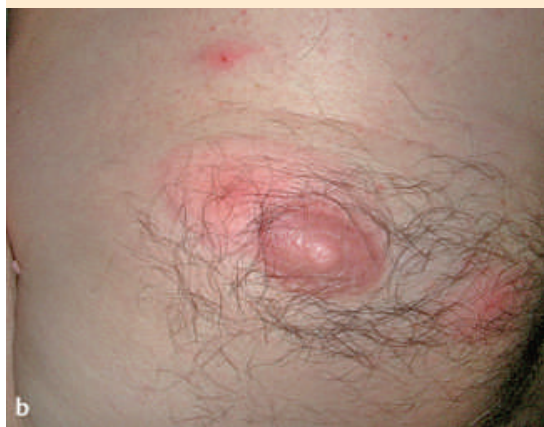

Bemerkenswert bei dem von uns betreuten Patienten war, dass es im Verlaufe eines Jahres zu zwei Rezidiven kam und der private Pool erst relativ spät als wahrscheinlichste Keimquelle erkannt wurde.

\section{Abstract}

\section{Whirlpool Dermatitis - A Special Type of Gram-Negative Folliculitis \\ $\nabla$}

We describe the case of a 45-year-old man with folliculitis due to Pseudomonas aeruginosa. During the following months there were two recurrences. Finally Pseudomonas aeruginosa was recovered from the private pool of the patient. In a short review of the literature some important aspects of whirlpool dermatitis due to Pseudomonas aeruginosa are described.

\section{Literatur}

1 Bhatia A, Brodell RT. „Hot tub folliculitis“. Test the waters - and the patient - for pseudomonas. Postgrad Med 1999; 106: 43 - 45

2 Burkhart CG, Shapiro R. Pseudomonas folliculitis. Development after home use of personal whirlpool spa. Cutis 1980; 25: $642-643$

3 Corbett $R$. Pseudomonas folliculitis from a spa pool in an immunocompromised patient. New Zeal Med J 1999; 112: 59

4 Crnich $\mathrm{Cl}$ et al. Hot tub-associated necrotizing pneumonia due to Pseudomonas aeruginosa. Clin Infect Dis 2003; 36: e55 - e57

5 Dockery G. Dermatology diagnosis: when a patient presents with a lower extremity rash. Podiatry Today 2006; 19: 30-34

6 Feder HM et al. Pseudomonas whirlpool dermatitis. Report of an outbreak in two families. Clin Pediatr 1983; 22: 638-642

7 Handrick W et al. Das „Hot-Foot-Syndrom“. Derm 2006; 12: 50-54

8 Handrick $W$, Nenoff $P$. Hot-Foot-Syndrom oder palmoplantare Hidradenitis? Akt Dermatol 2006; 32: 357 - 359

9 Hogan PA. Pseudomonas folliculitis. Austral J Dermatol 1997; 38: 93 94

10 Huminer $D$ et al. Home shower-bath pseudomonas folliculitis. Israel J Med Sci 1989; 25: 44-45

11 Kitamura $\mathrm{M}$ et al. Pseudomonas aeruginosa folliculitis: a sporadic case from use of a contaminated sponge. Br J Dermatol 1998; 139: 359_ 360

12 Maniatis AN et al. Pseudomonas aeruginosa folliculitis due to non- 0 : 11 serogroups: acquisition through use of contaminated synthetic sponges. Clin Infect Dis 1995; 21: 437-439

13 Rasmussen JE, Graves WH. Pseudomonas aeruginosa, hot tubs, and skin infections. Am J Dis Child 1982; 136: 553-554

14 Schille R, Handrick W. Whirlpool-Dermatitis bei Kindern. Sozialpädiatrie 1998; 20: $288-290$

15 Shirtcliffe P, Robinson GM. A case of severe pseudomonas folliculitis from a spa pool. New Zeal Med J 1998; 111: 389

16 Silverman AR, Nieland ML. Hot tub dermatitis: A familial outbreak of pseudomonas folliculitis. J Am Acad Dermatol 1983; 8: 153-156

17 Stähelin-Massik J et al. Pseudomonas folliculitis in a young child. Pediatr Infect Dis J 2000; 19: 362 - 363

18 Usatine RP. Itchy bumps on the back. West J Med 2000; 172: 366 - 367

19 Zacherle BJ, Silver DS. Hot tub folliculitis: a clinical syndrome. West J Med 1982; 137: 191 - 194

20 Zichichi $L$ et al. Pseudomonas aeruginosa folliculitis after shower/bath exposure. Int J Dermatol 2000; 39: 270-273 